\title{
Chiral partner structure of light nucleons in an extended parity doublet model
}

\author{
Takahiro Yamazaki ${ }^{*}$ and Masayasu Harada ${ }^{\dagger}$ \\ Department of Physics, Nagoya University, Nagoya 464-8602, Japan
}

(Received 20 September 2018; published 20 February 2019)

\begin{abstract}
We study chiral partner structure of four light nucleons, $N(939), N(1440), N(1535)$ and $N(1650)$, using an effective chiral model based on the parity doublet structure. In our model we introduce four chiral representations, $(1,2),(2,1),(2,3)$ and $(3,2)$, under $\mathrm{SU}(2)_{L} \otimes \mathrm{SU}(2)_{R}$ symmetry. We determine the model parameters by fitting them to available experimental values of masses, widths and the axial charge of $N(939)$ together with the axial charges of $N(1535)$ and $N(1650)$ by lattice analyses. We find five groups of solutions: In a group the chiral partner to $N(939)$ is $N(1440)$ having small chiral invariant mass. In another group, the chiral partner is a mixture of $N(1535)$ and $N(1650)$ having a large chiral invariant mass. Using the solutions, we give phenomenological predictions on the axial charges and changes of masses associated with chiral symmetry restoration.
\end{abstract}

DOI: 10.1103/PhysRevD.99.034012

\section{INTRODUCTION}

One of the most important features of QCD relevant to the low-energy hadron physics is the chiral symmetry and its spontaneous breaking. The spontaneous symmetry breaking generates mass differences between chiral partners as well as the mixing among different chiral representations. It is interesting to study the role of the chiral symmetry breaking to determine the properties and structures of baryons such as amount of the masses of baryons generated by the chiral symmetry breaking and the chiral partner of ground state nucleon.

In a hadronic model for light nucleons based onthe parity doublet structure [1-5] the chiral partner of $N(939)$ is $N(1535)$. One of the important features of the model is the existence of the chiral invariant mass denoted by $m_{0}$ which is not originated from the spontaneous chiral symmetry breaking. In other words, the mass splitting between $N(939)$ and its chiral partner $N(1535)$ is given by the spontaneous chiral symmetry breaking, which implies that the masses of $N(939)$ and $N(1535)$ approach $m_{0}$ when the chiral symmetry is restored.

This parity doublet structure is extended to include hyperons and/or excited nucleons such as $N(1440)$ and $N(1650)$ (see, e.g., Refs. [6-16]).

\footnotetext{
yamazaki@hken.phys.nagoya-u.ac.jp

'harada@hken.phys.nagoya-u.ac.jp
}

Published by the American Physical Society under the terms of the Creative Commons Attribution 4.0 International license. Further distribution of this work must maintain attribution to the author(s) and the published article's title, journal citation, and DOI. Funded by SCOAP .
Particularly, in Ref. [15], two $[(\mathbf{1}, \mathbf{2}) \oplus(\mathbf{2}, \mathbf{1})]$ representations under the chiral $\mathrm{SU}(2)_{L} \otimes \mathrm{SU}(2)_{R}$ are introduced to study $N(939), N(1440), N(1535)$ and $N(1650)$. Two types of solutions were found: In one type, the chiral partner of $N(939)$ is a mixture of $N(1535)$ and $N(1650)$ and two chiral invariant masses are almost $200 \mathrm{MeV}$. In another type, $N(939)$ and $N(1535)$ are chiral partners to each other and two chiral invariant masses are about $1000 \mathrm{MeV}$. On the other hand, in Ref. [14], a model is constructed in the three-flavor framework to further introduce the chiral $[(\mathbf{3}, \mathbf{6}) \oplus(\mathbf{6}, \mathbf{3})]$ representations under the chiral $\mathrm{SU}(3)_{L} \otimes \mathrm{SU}(3)_{R}$ symmetry, which corresponds to the chiral $[(\mathbf{2}, \mathbf{3}) \oplus(\mathbf{3}, \mathbf{2})]$ representations under the chiral $\mathrm{SU}(2)_{L} \otimes \mathrm{SU}(2)_{R}$ symmetry. It was shown that the $N(939)$ is dominated by the $[(\mathbf{3}, \mathbf{6}) \oplus(\mathbf{6}, \mathbf{3})]$ to reproduce the axial charge of nucleon as pointed in Ref. [8], and that the chiral invariant mass of the $N(939)$ is about $800 \mathrm{MeV}$. The large value of the chiral invariant mass of $N(939)$ seems consistent with the results by the lattice QCD analysis in Refs. [17,18], which shows that is almost constant even if temperature is increased.

In this paper, we make a general analysis using a twoflavor parity doublet model including chiral $[(\mathbf{1}, \mathbf{2}) \oplus$ $(\mathbf{2}, \mathbf{1})]$ and $[(\mathbf{2}, \mathbf{3}) \oplus(\mathbf{3}, \mathbf{2})]$ representations under the chiral $\mathrm{SU}(2)_{L} \otimes \mathrm{SU}(2)_{R}$ symmetry, with including derivative interactions to the pion fields. We will show that there exist five groups of solutions distinguishable by chiral invariant masses and mixing rates of nucleons. In a group of solutions, the chiral partner of $N(939)$ is $N(1440)$ having small chiral invariant mass of about $100 \mathrm{MeV}$ and $N(939)$ is dominated by the $[(\mathbf{2}, \mathbf{3}) \oplus(\mathbf{3}, \mathbf{2})]$ representation. In another group, on the other hand, $N(939)$ belongs dominantly to the $[(\mathbf{1}, \mathbf{2}) \oplus(\mathbf{2}, \mathbf{1})]$ representation having a large 
chiral invariant mass, and the chiral partner of $N(939)$ is a mixture of $N(1535)$ and $N(1650)$. Furthermore, we give predictions of axial charges, which could be checked in future experiments and/or lattice analyses. We also show changes of nucleon masses when the vacuum expectation value of $\sigma$, which is an order parameter of the spontaneous chiral symmetry breaking, is changed.

This paper is organized as follows: In Sec. II, we construct an extended model with parity doublet structure. Section III is a main part, where we show the numerical results of fitting on the chiral invariant masses and chiral partner structure. In Sec. IV, we provide our predictions on the axial charges and the changes of nucleon masses. Finally we will give a brief summary and discussions in Sec. V.

\section{AN EXTENDED PARITY DOUBLET MODEL}

In this section we introduce four baryon fields with parity doublet structure and construct a Lagrangian for baryons and scalar and pseudoscalar mesons based on the $\mathrm{SU}(2)_{L} \otimes \mathrm{SU}(2)_{R}$ chiral symmetry.

\section{A. Model construction}

The chiral representations of quarks under $\mathrm{SU}(2)_{L} \otimes$ $\mathrm{SU}(2)_{R}$ are written as

$$
q_{L} \sim(\mathbf{2}, \mathbf{1}), \quad q_{R} \sim(\mathbf{1}, \mathbf{2}),
$$

where the 2 and 1 in the above bracket express doublet and singlet, respectively. Since baryons are expressed as direct products of three quarks, we have the following possibilities for the chiral representations of baryons:

$$
\begin{aligned}
q \otimes q \otimes q & \sim[(\mathbf{2}, \mathbf{1}) \oplus(\mathbf{1}, \mathbf{2})]^{3} \\
& \sim 5[(\mathbf{2}, \mathbf{1}) \oplus(\mathbf{1}, \mathbf{2})] \oplus 3[(\mathbf{3}, \mathbf{2}) \oplus(\mathbf{2}, \mathbf{3})] \\
& \oplus[(\mathbf{4}, \mathbf{1}) \oplus(\mathbf{1}, \mathbf{4})] .
\end{aligned}
$$

After the chiral symmetry is spontaneously broken down to the flavor symmetry, nucleons appear from the representations of $(\mathbf{2}, \mathbf{1}) \oplus(\mathbf{1}, \mathbf{2})$ and $(\mathbf{3}, \mathbf{2}) \oplus(\mathbf{2}, \mathbf{3})$. In this paper we introduce two baryon fields corresponding to these two representations:

$$
\begin{array}{rlrl}
\psi_{1 l} & \sim(\mathbf{2}, \mathbf{1}), & & \psi_{1 r} \sim(\mathbf{1}, \mathbf{2}) \\
\psi_{2 l} \sim(\mathbf{1}, \mathbf{2}), & & \psi_{2 r} \sim(\mathbf{2}, \mathbf{1}) \\
\eta_{1 l} & \sim(\mathbf{2}, \mathbf{3}), & & \eta_{1 r} \sim(\mathbf{3}, \mathbf{2}) \\
\eta_{2 l} & \sim(\mathbf{3}, \mathbf{2}), & & \eta_{2 r} \sim(\mathbf{2}, \mathbf{3}) .
\end{array}
$$

Here the subscripts $l$ and $r$ express the chirality:

$$
\begin{aligned}
\gamma_{5} \psi_{i l} & =-\psi_{i l}, & & \gamma_{5} \psi_{i r}=+\psi_{i r} \\
\gamma_{5} \eta_{i l} & =-\eta_{i l}, & & \gamma_{5} \eta_{i r}=+\eta_{i r}
\end{aligned}
$$

for $i=1,2$.

For clarifying the representations under the chiral symmetry, we explicitly write the superscripts of the baryon fields as

$$
\begin{gathered}
\left(\psi_{1 l}\right)^{a},\left(\psi_{1 r}\right)^{\alpha},\left(\psi_{2 l}\right)^{\alpha},\left(\psi_{2 r}\right)^{a} \\
\eta_{1 l}^{(a, \alpha \beta)}, \eta_{1 r}^{(a b, \alpha)}, \eta_{2 l}^{(a b, \alpha)}, \eta_{2 r}^{(a, \alpha \beta)},
\end{gathered}
$$

where $a, b=1,2$ are for $\mathrm{SU}(2)_{L}$ and $\alpha, \beta=1,2$ for $\mathrm{SU}(2)_{R}$. Note that the superscripts $a b$ and $\alpha \beta$ of $\eta$ fields are symmetrized to express 3 representation: e.g., $\eta_{1 l}^{(a, \alpha \beta)}=\eta_{1 l}^{(a, \beta \alpha)}$. These fields transform under chiral $\mathrm{SU}(2)_{L} \otimes \mathrm{SU}(2)_{R}$ symmetry as

$$
\begin{gathered}
\left(\psi_{1 l(2 r)}\right)^{a} \rightarrow g_{L}^{a b}\left(\psi_{1 l(2 r)}\right)^{a}, \\
\left(\psi_{1 r(2 l)}\right)^{\alpha} \rightarrow g_{R}^{\alpha \beta}\left(\psi_{1 l(2 r)}\right)^{\alpha}, \\
\left(\eta_{1 l(2 r)}\right)^{(a, \alpha \beta)} \rightarrow g_{L}^{a b} g_{R}^{\alpha \gamma} g_{R}^{\beta \delta}\left(\eta_{1 l(2 r)}\right)^{(a, \alpha \beta),} \\
\left(\eta_{1 r(2 l)}\right)^{(a b, \alpha)} \rightarrow g_{L}^{a c} g_{L}^{b d} g_{R}^{\alpha \beta}\left(\eta_{1 r(2 l)}\right)^{(c d, \beta),}
\end{gathered}
$$

where $a, b, c, d=1,2$ are for $\mathrm{SU}(2)_{L}$ and $\alpha, \beta, \gamma, \delta=1,2$ for $\mathrm{SU}(2)_{R}$. The transformation properties under the parity and the charge conjugation are defined as

$$
\begin{aligned}
& \Psi_{1 l, 1 r} \underset{P}{\rightarrow} \gamma_{0} \Psi_{1 r, 1 l}, \quad \Psi_{2 l, 2 r} \underset{P}{\rightarrow}-\gamma_{0} \Psi_{2 r, 2 l}, \\
& \Psi_{1 l, 1 r} \underset{C}{\rightarrow} C\left(\bar{\Psi}_{1 r, 1 l}\right)^{T}, \quad \Psi_{2 l, 2 r} \underset{C}{\rightarrow}-C\left(\bar{\Psi}_{2 r, 2 l}\right)^{T},
\end{aligned}
$$

where $C=i \gamma^{2} \gamma^{0}$ and $\Psi=\psi, \eta$. The covariant derivatives for the fields are expressed as

$$
\begin{aligned}
& D_{\mu} \psi_{1 r, 2 l}=\left(\partial_{\mu}-i \mathcal{R}_{\mu}\right) \psi_{1 r, 2 l}, \\
& D_{\mu} \psi_{1 l, 2 r}=\left(\partial_{\mu}-i \mathcal{L}_{\mu}\right) \psi_{1 l, 2 r},
\end{aligned}
$$

and

$$
\begin{aligned}
\left(D_{\mu} \eta_{1 l, 2 r}\right)^{(a, \alpha \beta)}= & \partial_{\mu} \eta_{1 l, 2 r}^{(a, \alpha \beta)}-i\left(\mathcal{L}_{\mu}\right)_{b}^{a} \eta_{1 l, 2 r}^{(b, \alpha \beta)} \\
& -i\left[\left(\mathcal{R}_{\mu}\right)_{\rho}^{\alpha} \delta_{\sigma}^{\beta}+\delta_{\rho}^{\alpha}\left(\mathcal{R}_{\mu}\right)_{\sigma}^{\beta}\right] \eta_{1 l, 2 r}^{(a, \rho \sigma)}, \\
\left(D_{\mu} \eta_{1 r, 2 l}\right)^{(a b, \alpha)}= & \partial_{\mu} \eta_{1 r, 2 l}^{(a b, \alpha)}-i\left(\mathcal{R}_{\mu}\right)_{\beta}^{\alpha} \eta_{1 r, 2 l}^{(a b, \beta)} \\
& -i\left[\left(\mathcal{L}_{\mu}\right)_{c}^{a} \delta_{d}^{b}+\delta_{c}^{a}\left(\mathcal{L}_{\mu}\right)_{d}^{b}\right] \eta_{1 r, 2 l}^{(c d, a)},
\end{aligned}
$$

where $\mathcal{L}_{\mu}$ and $\mathcal{R}_{\mu}$ are the external gauge fields introduced by gauging the chiral $\mathrm{SU}(2)_{L} \otimes \mathrm{SU}(2)_{R}$ symmetry.

Next we introduce a $2 \times 2$ matrix field $M$ expressing scalar and pseudoscalar mesons made of a quark and an 
antiquark. The representation under $\mathrm{SU}(2)_{L} \otimes \mathrm{SU}(2)_{R}$ of the $M$ is

$$
M=\frac{\sigma}{2}+i \vec{\pi} \cdot \vec{T} \sim(\mathbf{2}, \mathbf{2}) .
$$

The transformation properties under the parity and the charge conjugation are given by

$$
M \underset{P}{\rightarrow} M^{\dagger}, \quad M \underset{C}{\rightarrow} M^{T} .
$$

The covariant derivative for $M$ is expressed as

$$
\left(D_{\mu} M\right)_{\alpha}^{a}=\partial_{\mu} M_{\alpha}^{a}-i\left(\mathcal{L}_{\mu}\right)_{b}^{a} M_{\alpha}^{b}+i M_{\beta}^{a}\left(\mathcal{R}_{\mu}\right)_{\alpha}^{\beta}
$$

Using the fields introduced above we construct a Lagrangian invariant under the chiral $\mathrm{SU}(2)_{L} \otimes \mathrm{SU}(2)_{R}$ symmetry.

Let us first consider terms including only $\psi_{1}$ and $\psi_{2}$ and their Yukawa interaction to the $M$ field. In the present analysis, we include interaction terms with one $M$ field. We expect that terms with more than one $M$ field are suppressed. Then, possible terms are expressed as

$$
\begin{aligned}
\mathcal{L}^{(1)}= & \bar{\psi}_{1 l} i \not \psi_{1 l}+\bar{\psi}_{1 r} i \not \psi_{1 r}+\bar{\psi}_{2 l} i \not \psi_{2 l}+\bar{\psi}_{2 r} i \not \psi_{2 r}-g_{1}\left(\bar{\psi}_{1 l} M \psi_{1 r}+\bar{\psi}_{1 r} M^{\dagger} \psi_{1 l}\right)-g_{2}\left(\bar{\psi}_{2 r} M \psi_{2 l}+\bar{\psi}_{2 l} M^{\dagger} \psi_{2 r}\right) \\
& -m_{0}^{(1)}\left(\bar{\psi}_{1 l} \psi_{2 r}-\bar{\psi}_{1 r} \psi_{2 l}-\bar{\psi}_{2 l} \psi_{1 r}+\bar{\psi}_{2 r} \psi_{1 l}\right) .
\end{aligned}
$$

A part including $\eta_{1}$ and $\eta_{2}$ with one $M$ is

$$
\begin{aligned}
\mathcal{L}^{(2)}= & \left(\bar{\eta}_{1 l}\right)_{(a, \alpha \beta)} i \not D\left(\eta_{1 l}\right)^{(a, \alpha \beta)}+\left(\bar{\eta}_{1 r}\right)_{(a b, \alpha)} i \not D\left(\eta_{1 r}\right)^{(a b, \alpha)}+\left(\bar{\eta}_{2 l}\right)_{(a b, \alpha)} i \not D\left(\eta_{2 l}\right)^{(a b, \alpha)}+\left(\bar{\eta}_{2 r}\right)_{(a, \alpha \beta)} i \not D\left(\eta_{2 r}\right)^{(a, \alpha \beta)} \\
& -g_{3}\left[\left(\bar{\eta}_{1 r}\right)_{(a b, \alpha)}(M)_{\beta}^{a}\left(\eta_{1 l}\right)^{(b, \alpha \beta)}+\left(\bar{\eta}_{1 l}\right)_{(a, \alpha \beta)}\left(M^{\dagger}\right)_{b}^{\alpha}\left(\eta_{1 r}\right)^{(a b, \beta)}\right] \\
& -g_{4}\left[\left(\bar{\eta}_{2 l}\right)_{(a b, \alpha)}(M)_{\beta}^{a}\left(\eta_{2 r}\right)^{(b, \alpha \beta)}+\left(\bar{\eta}_{2 r}\right)_{(a, \alpha \beta)}\left(M^{\dagger}\right)_{b}^{\alpha}\left(\eta_{2 l}\right)^{(a b, \beta)}\right] \\
& -m_{0}^{(2)}\left[\left(\bar{\eta}_{1 l}\right)_{(a, \alpha \beta)}\left(\eta_{2 r}\right)^{(a, \alpha \beta)}-\left(\bar{\eta}_{1 r}\right)_{(a b, \alpha)}\left(\eta_{2 l}\right)^{(a b, \alpha)}+\left(\bar{\eta}_{2 r}\right)_{(a, \alpha \beta)}\left(\eta_{1 l}\right)^{(a, \alpha \beta)}-\left(\bar{\eta}_{2 l}\right)_{(a b, \alpha)}\left(\eta_{1 r}\right)^{(a b, \alpha)}\right] .
\end{aligned}
$$

Yukawa interaction terms connecting $\psi$ fields to $\eta$ fields with one $M$ field are expressed as

$$
\begin{aligned}
\mathcal{L}^{(3)}= & -y_{1}\left[\epsilon_{b c}\left(\bar{\psi}_{1 r}\right)_{\alpha}(M)_{\beta}^{b}\left(\eta_{1 l}\right)^{(c, \alpha \beta)}+\epsilon_{\beta \sigma}\left(\bar{\psi}_{1 l}\right)_{a}\left(M^{\dagger}\right)_{b}^{\beta}\left(\eta_{1 r}\right)^{(a b, \sigma)}+\epsilon^{b c}\left(\bar{\eta}_{1 l}\right)_{(c, \alpha \beta)}\left(M^{\dagger}\right)_{b}^{\beta}\left(\psi_{1 r}\right)^{\alpha}+\epsilon^{\beta \sigma}\left(\bar{\eta}_{1 r}\right)_{a b, \sigma}(M)_{\beta}^{b}\left(\psi_{1 l}\right)^{a}\right] \\
& -y_{2}\left[\epsilon_{\beta \sigma}\left(\bar{\psi}_{2 r}\right)_{a}\left(M^{\dagger}\right)_{b}^{\beta}\left(\eta_{2 l}\right)^{(a b, \sigma)}+\epsilon_{b c}\left(\bar{\psi}_{2 l}\right)_{\alpha}(M)_{\beta}^{b}\left(\eta_{2 r}\right)^{(c, \alpha \beta)}+\epsilon^{\beta \sigma}\left(\bar{\eta}_{2 l}\right)_{(a b, \sigma)}(M)_{\beta}^{b}\left(\psi_{2 r}\right)^{a}+\epsilon^{b c}\left(\bar{\eta}_{2 r}\right)_{(c, \alpha \beta)}\left(M^{\dagger}\right)_{b}^{\beta}\left(\psi_{2 l}\right)^{\alpha}\right] .
\end{aligned}
$$

In addition to the nonderivative interactions shown above, we need to include derivative interactions. Possible interaction terms including one derivative are given by

$$
\begin{aligned}
\mathcal{L}^{(4)}= & \frac{i}{f_{\pi}}\left(-a_{1}\left[\bar{\psi}_{1 l} \not D M \psi_{2 l}-\bar{\psi}_{1 r} \not D M^{\dagger} \psi_{2 r}+\bar{\psi}_{2 l} \not D M^{\dagger} \psi_{1 l}-\bar{\psi}_{2 r} \not D M \psi_{1 r}\right]\right. \\
& -a_{2}\left[\bar{\eta}_{1 r(a b, \alpha)}(\not D M)_{\beta}^{a} \eta_{2 r}^{(b, \alpha \beta)}-\bar{\eta}_{1 l(a, \alpha \beta)}\left(\not D M^{\dagger}\right)_{b}^{\alpha} \eta_{2 r}^{(a b, \beta)}+\bar{\eta}_{2 r(a, \alpha \beta)}\left(\not D M^{\dagger}\right)_{b}^{\alpha} \eta_{1 r}^{(a b, \beta)}-\bar{\eta}_{2 l(a b, \alpha)}(\not D M)_{\beta}^{a} \eta_{1 l}^{(b, \alpha \beta)}\right] \\
& -a_{3}\left[\epsilon_{a b} \bar{\psi}_{1 r \alpha}(\not D M)_{\beta}^{a} \eta_{2 r}^{(b, \alpha \beta)}-\epsilon_{\alpha \beta} \bar{\psi}_{1 l a}\left(\not D M^{\dagger}\right)_{b}^{\alpha} \eta_{2 l}^{(a b, \beta}+\epsilon^{a b} \bar{\eta}_{2 r(b, \alpha \beta)}\left(\not D M^{\dagger}\right)_{a}^{\beta} \psi_{1 r}^{\alpha}-\epsilon^{\alpha \beta} \bar{\eta}_{2 l(a b, \beta)}(D M)_{\alpha}^{b} \psi_{1 l}^{a}\right] \\
& \left.-a_{4}\left[\epsilon_{\alpha \beta} \bar{\psi}_{2 r a}\left(\not D M^{\dagger}\right)_{b}^{\alpha} \eta_{1 r}^{(a b, \beta)}-\epsilon_{a b} \bar{\psi}_{2 l \alpha}(D M)_{\beta}^{a} \eta_{1 l}^{(\alpha \beta, b)}+\epsilon^{\alpha \beta} \bar{\eta}_{1 r(a b, \beta)}(\not D M)_{\alpha}^{b} \psi_{2 r}^{a}-\epsilon^{a b} \bar{\eta}_{1 l(\alpha \beta, b)}\left(\not D M^{\dagger}\right)_{a}^{\beta} \psi_{2 l}^{\alpha}\right]\right) .
\end{aligned}
$$

Combining the above terms together, the Lagrangian in the present analysis is given by

$$
\mathcal{L}=\mathcal{L}^{(1)}+\mathcal{L}^{(2)}+\mathcal{L}^{(3)}+\mathcal{L}^{(4)}+\mathcal{L}_{\text {meson }}
$$

where the mesonic part $\mathcal{L}_{\text {meson }}$ is written as

$$
\mathcal{L}_{\text {meson }}=\operatorname{Tr}\left[D_{\mu} M \cdot D^{\mu} M^{\dagger}\right]-V(M),
$$

where $V(M)$ is a meson potential term. In this paper, we do not specify the form of the potential, but we assume that this potential provides the vacuum expectation value (VEV) of $M$ as $\langle M\rangle=\operatorname{diag}\left(f_{\pi} / 2, f_{\pi} / 2\right)$, where $f_{\pi}$ is the pion decay constant. 


\section{B. Mass matrix}

We have constructed the Lagrangian by requiring the chiral $\mathrm{SU}(2)_{L} \otimes \mathrm{SU}(2)_{R}$ invariance. To study the properties of nucleons, we decompose baryons in the chiral representation to irreducible representations of the flavor symmetry as

$$
\begin{gathered}
\psi_{1 l, 2 r}=N_{1 l, 2 r}^{(1)}, \quad \psi_{1 r, 2 l}=N_{1 r, 2 l}^{(1)}, \\
\eta_{1 l, 2 r}^{(a, \alpha \beta)}=\Delta_{1 l, 2 r}^{a \alpha \beta}+\frac{1}{\sqrt{6}}\left(\epsilon^{\alpha a} \delta_{k}^{\beta}+\epsilon^{\beta a} \delta_{k}^{\alpha}\right)\left(N_{1 l, 2 r}^{(2)}\right)^{k}, \\
\eta_{1 r, 2 l}^{(a b, \alpha)}=\Delta_{1 r, 2 l}^{a b \alpha}+\frac{1}{\sqrt{6}}\left(\epsilon^{a \alpha} \delta_{k}^{b}+\epsilon^{b \alpha} \delta_{k}^{a}\right)\left(N_{1 r, 2 l}^{(2)}\right)^{k} .
\end{gathered}
$$

In the following, for convenience we redefine the nucleon fields as

$$
N_{1}^{(i)}=N_{1}^{(i)}, \quad N_{2}^{\prime(i)}=\gamma_{5} N_{2}^{(i)}, \quad(i=1,2) .
$$

The mass terms for the redefined fields are expressed as

$$
-\bar{N}^{\prime} M_{N}^{\prime} N^{\prime}
$$

with

$$
N^{\prime T} \equiv\left(N_{1}^{\prime(1)}, N_{1}^{\prime(2)} N_{2}^{\prime(1)} N_{2}^{\prime(2)}\right)
$$

and

$$
M_{N}^{\prime}=\left(\begin{array}{cccc}
\frac{g_{1}}{2} f_{\pi} & -\frac{3 y_{1}}{2 \sqrt{6}} f_{\pi} & m_{0}^{(1)} & 0 \\
-\frac{3 y_{1}}{2 \sqrt{6}} f_{\pi} & \frac{g_{3}}{4} f_{\pi} & 0 & m_{0}^{(2)} \\
m_{0}^{(1)} & 0 & -\frac{g_{2}}{2} f_{\pi} & \frac{3 y_{2}}{2 \sqrt{6}} f_{\pi} \\
0 & m_{0}^{(2)} & \frac{3 y_{2}}{2 \sqrt{6}} f_{\pi} & -\frac{g_{4}}{4} f_{\pi}
\end{array}\right) .
$$

The mass eigenstates denoted by

$$
N_{\text {phys }}^{\prime T} \equiv\left({N_{+}^{\prime}}_{+}^{(1)},{N_{+}^{\prime}}_{+}^{(2)}, N_{-}^{\prime(1)}, N_{-}^{\prime(2)}\right)_{\text {phys }}
$$

are obtained by diagonalizing the above mass matrix $M_{N}^{\prime}$. We note that parities of all fields in $N^{\prime}$ are even due to the redefinition given in Eq. (26). Two eigenvalues of the mass matrix $M_{N}^{\prime}$ are negative in our analysis, and we regard the parities of these states as negative.

\section{One pion interactions and axial charges}

The interaction terms of nucleons to one pion are given from the Lagrangian as

$$
\bar{N}^{\prime} C_{\pi N N}^{\prime} i \gamma_{5} \pi N^{\prime}+\bar{N}^{\prime} C_{\partial \pi N N}^{\prime} \gamma^{\mu} \partial_{\mu} \pi \gamma_{5} N^{\prime},
$$

where $\pi=\vec{\pi} \cdot \vec{\tau}$ and

$$
\begin{gathered}
C_{\pi N N}^{\prime}=\left(\begin{array}{cccc}
-\frac{g_{1}}{2} & -\frac{y_{1}}{2 \sqrt{6}} & 0 & 0 \\
-\frac{y_{1}}{2 \sqrt{6}} & \frac{5 g_{3}}{12} & 0 & 0 \\
0 & 0 & -\frac{g_{2}}{2} & -\frac{y_{2}}{2 \sqrt{6}} \\
0 & 0 & -\frac{y_{2}}{2 \sqrt{6}} & \frac{5 g_{4}}{12}
\end{array}\right), \\
C_{\partial \pi N N}^{\prime}=\left(\begin{array}{cccc}
0 & 0 & -\frac{a_{1}}{2 f_{\pi}} & -\frac{a_{3}}{2 \sqrt{6} f_{\pi}} \\
0 & 0 & -\frac{a_{4}}{2 \sqrt{6} f_{\pi}} & \frac{5 a_{2}}{12 f_{\pi}} \\
-\frac{a_{1}}{2 f_{\pi}} & -\frac{a_{4}}{2 \sqrt{6} f_{\pi}} & 0 & 0 \\
-\frac{a_{3}}{2 \sqrt{6} f_{\pi}} & \frac{5 a_{2}}{12 f_{\pi}} & 0 & 0
\end{array}\right) .
\end{gathered}
$$

The axial-vector charge matrix is determined as

$$
\bar{N}^{\prime} G_{A}^{\prime} \gamma^{\mu} \mathcal{A}_{\mu} \gamma_{5} N^{\prime}
$$

where

$$
G_{A}^{\prime}=\left(\begin{array}{cccc}
-1 & 0 & -\frac{a_{1} \sigma_{0}}{f_{\pi}} & -\frac{a_{3} \sigma_{0}}{\sqrt{6} f_{\pi}} \\
0 & \frac{5}{3} & -\frac{a_{4} \sigma_{0}}{\sqrt{6} f_{\pi}} & \frac{5 a_{2} \sigma_{0}}{6 f_{\pi}} \\
-\frac{a_{1} \sigma_{0}}{f_{\pi}} & -\frac{a_{4} \sigma_{0}}{\sqrt{6} f_{\pi}} & 1 & 0 \\
-\frac{a_{3} \sigma_{0}}{\sqrt{6} f_{\pi}} & \frac{5 a_{2} \sigma_{0}}{6 f_{\pi}} & 0 & -\frac{5}{3}
\end{array}\right) .
$$

In the present analysis, we identify the mass eigenstates as

$N_{\text {phys }}^{T} \equiv(N(939), N(1440), N(1535), N(1650))_{\text {phys }}$.

\section{CHIRAL INVARIANT MASSES AND PARTNER STRUCTURE}

In this section we determine the values of model parameters and study the mixing structure of relevant baryons.

As we said in the previous section, we set the VEV of $\sigma$ to be the pion decay constant:

$$
\sigma_{0}=f_{\pi}=92.4 \mathrm{MeV}
$$

Beside this parameter, there are twelve parameters in this model:

$$
m_{0}^{(1)}, m_{0}^{(2)}, g_{1}, g_{2}, g_{3}, g_{4}, y_{1}, y_{2}, a_{1}, a_{2}, a_{3}, a_{4} .
$$

We list values of relevant physical quantities determined from experiments and lattice analyses in Table I. Among them, we use the following 10 physical values as inputs: nucleon masses: 
TABLE I. Experimental values of masses and partial decay widths of baryons listed in Ref. [19]. The column indicated by $P= \pm$ shows the parity of the nucleon. Unit of masses and widths is $\mathrm{MeV}$. The error of $m_{N(939)}$ expresses the mass difference between the proton and neutron. [lat] indicates that the value is obtained by the lattice analysis in Ref. [20].

\begin{tabular}{lcccc}
\hline \hline & $P$ & Mass & Width $\left[\Gamma_{N^{*} \rightarrow N \pi}\right]$ & Axial charge \\
\hline $\mathrm{N}(939)$ & + & $939.0 \pm 1.3$ & $\ldots$ & $1.272 \pm 0.002$ \\
$\mathrm{~N}(1440)+$ & $1430 \pm 20$ & $228 \pm 74$ & $\ldots$ \\
$\mathrm{N}(1535)-$ & $1535 \pm 10$ & $68 \pm 19$ & $\mathcal{O}(0.1)$ [lat] \\
$\mathrm{N}(1650)-$ & $1655 \pm 15$ & $84 \pm 23[$ to $\mathrm{N}(939)]$ & 0.55 [lat] \\
\hline \hline
\end{tabular}

$$
\begin{aligned}
m_{N(939)} & =939 \mathrm{MeV}, \\
m_{N(1440)} & =1430 \mathrm{MeV}, \\
m_{N(1535)} & =1535 \mathrm{MeV}, \\
m_{N(1650)} & =1650 \mathrm{MeV},
\end{aligned}
$$

partial decay widths: ${ }^{1}$

$$
\begin{aligned}
\Gamma(N(1440) \rightarrow N(939)+\pi) & =228 \mathrm{MeV}, \\
\Gamma(N(1535) \rightarrow N(939)+\pi) & =68 \mathrm{MeV}, \\
\Gamma(N(1650) \rightarrow N(939)+\pi) & =84 \mathrm{MeV}, \\
\Gamma(N(1650) \rightarrow N(1440)+\pi) & =22 \mathrm{MeV},
\end{aligned}
$$

and axial charges:

$$
\begin{aligned}
& g_{A}(N(939))=1.272, \\
& g_{A}(N(1650)=0.55 .
\end{aligned}
$$

In addition to the above inputs, we use the following range of $g_{A}(N(1535))$ to restrict the parameters:

$$
-0.25 \leq g_{A}(N(1535)) \leq 0.25
$$

Furthermore, we restrict the parameters by requiring all the components of axial-charge matrix on the physical base are no larger than 5 .

In this analysis, we first fix the chiral invariant masses $m_{0}^{(1)}$ and $m_{0}^{(2)}$ to certain values of every $5 \mathrm{MeV}$ from 0 to $1500 \mathrm{MeV}$, and determine other ten parameters from the values shown in Eqs. (39)-(41). Here, $m_{0}^{(1)}$ and $m_{0}^{(2)}$ are chiral invariant masses of $[(\mathbf{1}, \mathbf{2}) \oplus(\mathbf{2}, \mathbf{1})]$ and $[(\mathbf{2}, \mathbf{3}) \oplus$ $(\mathbf{3}, \mathbf{2})$ ] representations, respectively.

We find that solutions are categorized into five groups as shown in Fig. 1. In group 1 indicated by purple + symbols, both chiral invariant masses are less than

\footnotetext{
${ }^{1}$ In calculating the decay widths, we use the pion mass as $m_{\pi}=137 \mathrm{MeV}$.
}

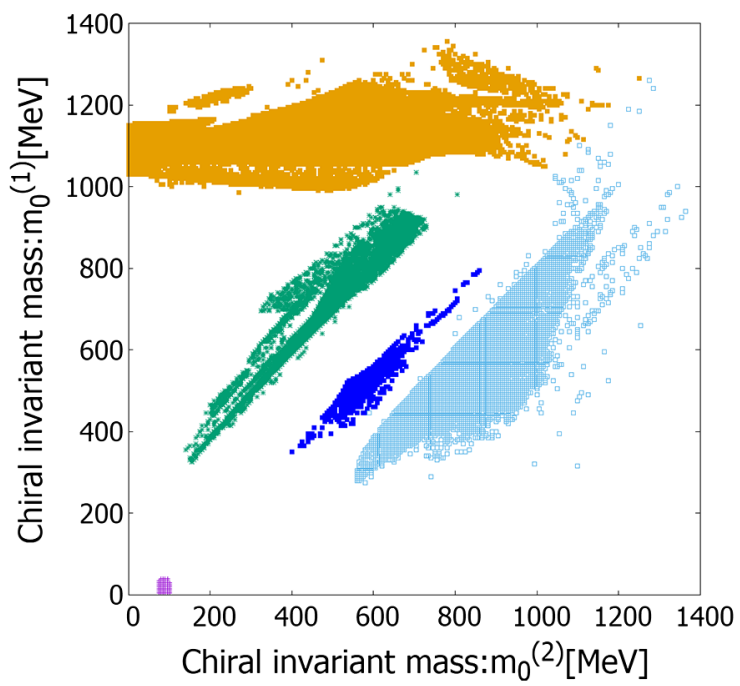

FIG. 1. Chiral invariant masses for which we find solutions to reproduce the values in Eqs. (39)-(41).

$100 \mathrm{MeV}$. In the range where $m_{0}^{(1)}$ is between 200 and $900 \mathrm{MeV}$, three lumps exist: group 2 indicated by blue - symbols; group 3 by light green $\times$ symbols; and group 4 light blue $\square$ symbols. They are characterized by

$$
\begin{aligned}
& m_{0}^{(1)} \sim m_{0}^{(2)}[\text { group 2], } \\
& m_{0}^{(1)} \geq m_{0}^{(2)}[\text { group } 3], \\
& m_{0}^{(1)}<m_{0}^{(2)}[\text { group 4], } \\
& m_{0}^{(1)} \geq m_{0}^{(2)}[\text { group 5]. }
\end{aligned}
$$

In group 5 indicated by yellow $\square$, the chiral invariant mass $m_{0}^{(1)}$ takes a large value of about $1000 \mathrm{MeV}$.

In Fig. 2, we show the mixing structure of nucleons: $N(939), N(1440), N(1535)$ and $N(1650)$ for group 1 to group 5. Here the horizontal axis shows the value of axial charge of $N(1535)$ and the vertical axis shows the percentages of $\psi_{1}$ indicated by magenta $\nabla$ symbols, $\eta_{1}$ by brown $\circ$ symbols, $\psi_{2}$ by green $\diamond$ symbols and $\eta_{2}$ by navy $\Delta$ symbols. In Table II, we summarize features of mixing rates for each group. The first row in Fig. 2 shows that the dominant component of $N(939)$ is $\eta_{1}$ indicated by brown $\circ$ belonging to $[(\mathbf{2}, \mathbf{3}) \oplus(\mathbf{3}, \mathbf{2})]$ representation. We note that we cannot find any solutions for $g_{A}(N(1535)) \gtrsim$ -0.1 in group 1 . One can easily see that $N(1440)$ is dominated by $\eta_{2}($ navy $\Delta), N(1535)$ by $\psi_{1}$ (magenta $\nabla$ ) belonging to $[(\mathbf{1}, \mathbf{2}) \oplus(\mathbf{2}, \mathbf{1})]$ representation and $N(1650)$ bv $\eta_{2}$ (green $\diamond$ ). Since $\eta_{1}$ and $\eta_{2}$ are chiral partners to each other, we conclude that $N(1440)$ dominated by $\eta_{2}$ is the chiral partner to $N(939)$ dominated by $\eta_{1}$. We would like to stress that this partner structure can be realized when 

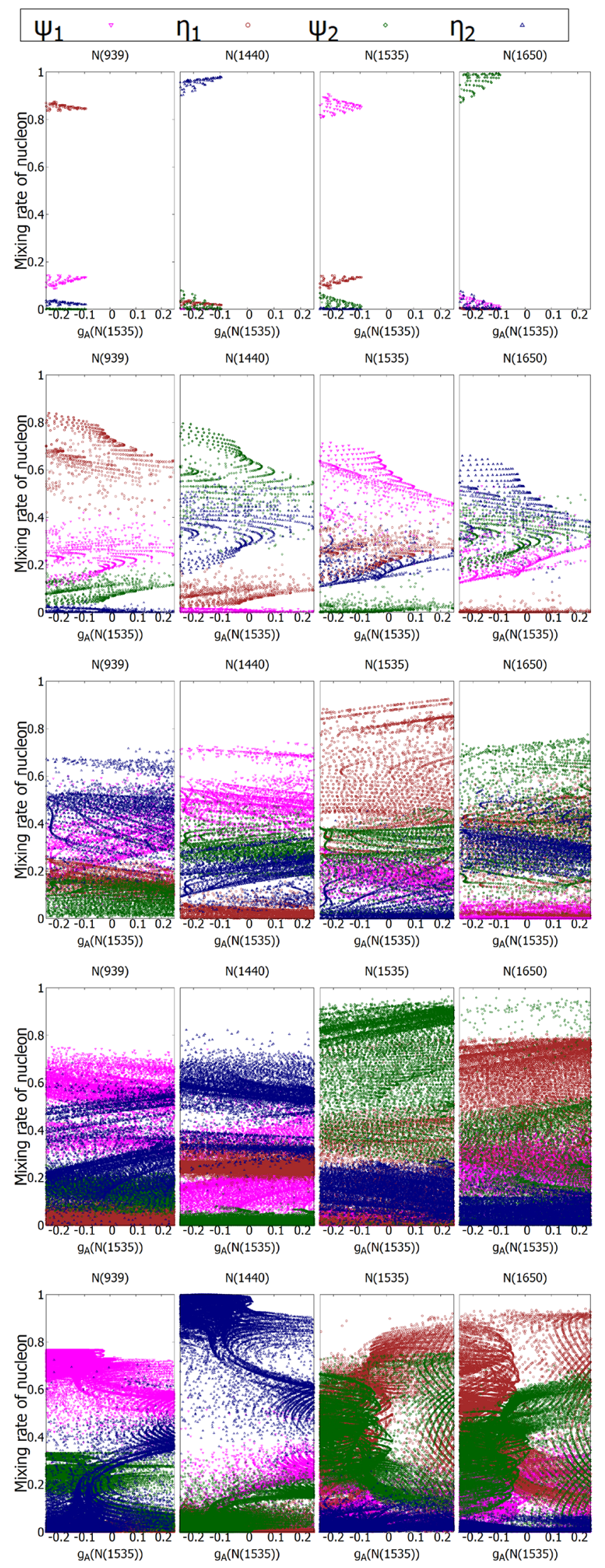

FIG. 2. Mixing rates of nucleons. The first to fifth rows show group 1 to group 5 . the chiral invariant masses of $N(939)$ and $N(1440)$ are small.

In group 2 (the second row of Fig. 2), $\eta_{1}$ (brown $\circ$ ) belonging to $[(\mathbf{2}, \mathbf{3}) \oplus(\mathbf{3}, \mathbf{2})]$ representation is a dominant component in the $N(939)$ and $\psi_{1}$ (magenta $\nabla$ ) almost occupies $N(1535)$, similarly to group 1 . A difference between group 1 and group 2 appears in the rate of $\psi_{2}$ (green $\diamond)$ in $N(1440)$. In group 1 , the mixing rate of $\psi_{2}$ in $N(1440)$ is smaller than 0.1 as can be seen in the first row of Fig. 2. On the other hand, the rate of $\psi_{2}$ is larger than 0.2 and $\psi_{2}$ is included in $N(1440)$ dominantly as shown in the second row of Fig. 2. Here the rate of $\eta_{2}$ component (navy $\Delta$ ) included in $N(1650)$ is high, but $N(1440)$ and $N(1535)$ include a certain amount of the $\eta_{2}$ component. So, it is difficult to identify the chiral partner of $N(939)$ in group 2 .

In group 3, group 4 and group 5, N(939) is composed of $\psi_{1}$ (magenta $\nabla$ ) or $\eta_{2}$ (navy $\Delta$ ) dominantly and negative parity nucleons, $N(1535)$ and $N(1650)$ have $\psi_{2}$ (green $\left.\diamond\right)$ or $\eta_{2}$ (brown $\circ$ ) mainly, as can be seen in the third, fourth and fifth rows in Fig. 2. This indicates that the chiral partner of $N(939)$ is a mixture of two negative parity nucleons in these groups, differently from group 1 and group 2. Table II shows that group 5 is distinguished from group 3 by the mixing rate of $\eta_{2}$ component in $N(1650)$ : The rate is larger than 0.1 in group 3 , while it is no greater than 0.1 in group 5. On the other hand, it is difficult to distinguish group 4 with group 5 and group 3 with group 4 by mixing rates. In the present work, we use the values of chiral invariant masses in addition to the mixing rates to separate these groups: The chiral invariant masses satisfy $m_{0}^{(1)}<m_{0}^{(2)}$ in group 4; group 3 is characterized by $m_{0}^{(1)} \geq$ $m_{0}^{(2)}$ and $\eta_{2}>0.1$ in $N(1650)$, while group 5 by $m_{0}^{(1)} \geq$ $m_{0}^{(2)}$ and $\eta_{2} \leq 0.1$ in $N(1650)$. In Ref. [15], two $[(\mathbf{1}, \mathbf{2}) \oplus$ $(\mathbf{2}, \mathbf{1})]$ representations are used to study $N(939), N(1440)$, $N(1535)$ and $N(1650)$. The authors found two types of solutions. In one type ("minimum 1" and "minimum 2"), the chiral partner of $N(939)$ is a mixture of $N(1535)$ and $N(1650)$ in vacuum and both chiral invariant masses are almost $200 \mathrm{MeV}$. In another type ("minimum 3") $N(939)$ and $N(1535)$ are chiral partners to each other and both chiral invariant masses are about $1000 \mathrm{MeV}$. In the present analysis, we find solutions for which $N(939)$ is dominated by $[(\mathbf{1}, \mathbf{2}) \oplus(\mathbf{2}, \mathbf{1})]$ representation in groups 3 , 4 and 5. Comparing mixing structure and the chiral invariant masses, we think that the minimum 1 and minimum 2 are consistent with group 3 and minimum 3 with group 4 or 5 . We show typical examples of mixing rates of nucleons in Table III.

In Ref. [14], $[(\mathbf{3}, \overline{\mathbf{3}}) \oplus(\overline{\mathbf{3}}, \mathbf{3})]$ and $[(\mathbf{3}, \mathbf{6}) \oplus(\mathbf{6}, \mathbf{3})]$ representations under the chiral $U(3)_{\mathrm{L}} \otimes U(3)_{\mathrm{R}}$ symmetry are introduced to study six nucleons including $N(939)$, $N(1440), N(1535)$ and $N(1650)$. This analysis indicates that $N(939)$ have $[(\mathbf{3}, \mathbf{6}) \oplus(\mathbf{6}, \mathbf{3})]$ dominantly and the 
TABLE II. Features of mixing rates. For example, in the column for group $1, \eta_{1}>0.8$ in the row of $N(939)$ implies that the percentage of $\eta_{1}$ component in $N(939)$ is always larger than 0.8 .

\begin{tabular}{lccccc}
\hline \hline Group color & Group 1 purple & Group 2 blue & Group 3 light green & Group 4 sky & Group 5 yellow \\
\hline$N(939)$ & $\eta_{1}>0.8$ & $\eta_{1}>0.35$ & $\eta_{1}<0.45$ & $\eta_{1}<0.35$ & $\eta_{1}<0.35$ \\
$N(1440)$ & $\psi_{1}<0.01$ & $\psi_{1}<0.025$ & $\psi_{1}>0.25$ & $\psi_{1}<0.75$ & $\psi_{1}<0.6$ \\
& $\psi_{2}<0.1$ & $\psi_{2}>0.2$ & $\psi_{2}>0.15$ & 0.2 & $\psi_{2}<0.35$ \\
$N(1650)$ & $\eta_{2}<0.1$ & $\eta_{2}>0.1$ & $\eta_{2}>0.1$ & $\eta_{2}<0.3$ & $\eta_{2} \leq 0.1$ \\
\hline \hline
\end{tabular}

TABLE III. Typical values of mixing rates of nucleons.

\begin{tabular}{lllllll}
\hline \hline Nucleon & & Group 1 & Group 2 & Group 3 & Group 4 & Group 5 \\
\hline$N(939)$ & $\psi_{1}$ & 0.113 & 0.21 & 0.518 & 0.677 & 0.768 \\
& $\eta_{1}$ & 0.856 & 0.735 & 0.098 & 0.032 & 0.001 \\
& $\psi_{2}$ & 0.002 & 0.052 & 0.133 & 0.163 & 0.227 \\
& $\eta_{2}$ & 0.029 & 0.002 & 0.251 & 0.128 & 0.004 \\
$N(1440)$ & $\psi_{1}$ & 0.002 & 0.003 & 0.355 & 0.092 & 0.002 \\
& $\eta_{1}$ & 0.029 & 0.043 & 0.021 & 0.223 & 0.041 \\
& $\psi_{2}$ & 0.033 & 0.627 & 0.368 & 0.01 & 0.001 \\
& $\eta_{2}$ & 0.936 & 0.327 & 0.256 & 0.675 & 0.956 \\
$N(1535)$ & $\psi_{1}$ & 0.819 & 0.641 & 0.126 & 0.012 & 0.137 \\
& $\eta_{1}$ & 0.109 & 0.222 & 0.801 & 0.212 & 0.322 \\
& $\psi_{2}$ & 0.068 & 0.007 & 0.057 & 0.615 & 0.528 \\
& $\eta_{2}$ & 0.004 & 0.130 & 0.015 & 0.161 & 0.013 \\
$N(1650)$ & $\psi_{1}$ & 0.066 & 0.146 & 0.001 & 0.218 & 0.093 \\
& $\eta_{1}$ & 0.006 & 0.0 & 0.08 & 0.533 & 0.636 \\
& $\psi_{2}$ & 0.897 & 0.314 & 0.442 & 0.212 & 0.244 \\
& $\eta_{2}$ & 0.031 & 0.540 & 0.477 & 0.037 & 0.027 \\
\hline \hline
\end{tabular}

chiral invariant mass is $500-800 \mathrm{MeV}$. This is consistent with group 2 in our analysis.

\section{PHENOMENOLOGICAL PREDICTIONS}

In the previous section, we showed the mixing structure of nucleons together with their chiral invariant masses. In this section, we present phenomenological predictions on the axial charges and the changes of nucleon masses against the change of VEV of $\sigma$.

\section{A. Axial charges}

We define transition axial charge as off-diagonal elements of the following axial-charge matrix on the physical base:

$$
\bar{N}_{\text {phys }}^{\prime} G_{\text {Aphys }}^{\prime} \gamma^{\mu} \mathcal{A}_{\mu} \gamma_{5} N_{\text {phys }}^{\prime},
$$

where

$$
G_{\text {Aphys }}^{\prime}=\left(\begin{array}{cccc}
g_{A}\left(N_{1}\right) & g_{A}\left(N_{1} N_{2}\right) & g_{A}\left(N_{1} N_{3}\right) & g_{A}\left(N_{1} N_{4}\right) \\
g_{A}\left(N_{1} N_{2}\right) & g_{A}\left(N_{2}\right) & g_{A}\left(N_{2} N_{3}\right) & g_{A}\left(N_{2} N_{4}\right) \\
g_{A}\left(N_{1} N_{3}\right) & g_{A}\left(N_{2} N_{3}\right) & g_{A}\left(N_{3}\right) & g_{A}\left(N_{3} N_{4}\right) \\
g_{A}\left(N_{1} N_{4}\right) & g_{A}\left(N_{2} N_{4}\right) & g_{A}\left(N_{3} N_{4}\right) & g_{A}\left(N_{4}\right)
\end{array}\right)
$$

and

$$
\left(N_{1}, N_{2}, N_{3}, N_{4}\right) \equiv\left(N_{+}^{\prime(1)} N_{+}^{\prime(2)} N_{-}^{\prime(1)} N_{-}^{\prime(2)}\right)_{\text {phys }} .
$$

In the present model, the following relation for the diagonal axial charges is satisfied:

$$
\sum_{i=1}^{4} g_{A}\left(N_{i}\right)=0
$$

Now we use axial charge: $g_{A}\left(N_{1}\right)=g_{A}(N(939))=1.272$ and $g_{A}\left(N_{4}\right)=g_{A}(N(1650))=0.55$ as input. So this relation is

$$
g_{A}\left(N_{2}\right)+g_{A}\left(N_{3}\right)=-1.822 .
$$

We plot this relation in Fig. 3. This plot shows that, when the axial charge of $N(1535)$ is in the range consistent with

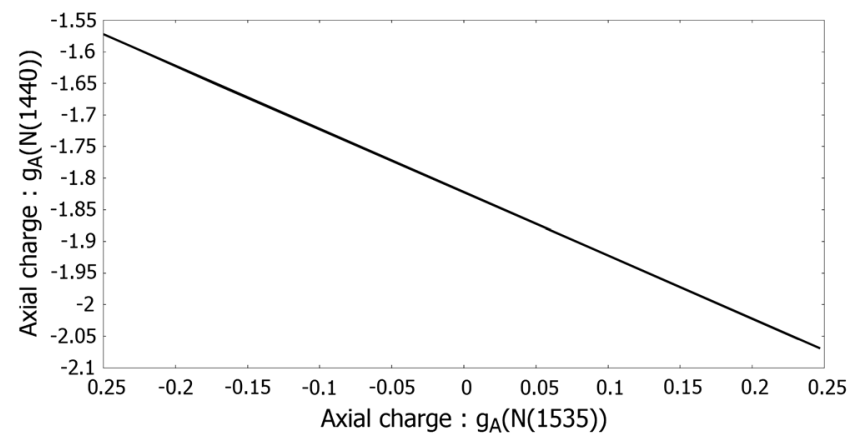

FIG. 3. The values of $g_{A}\left(N_{2}\right)$ and $g_{A}\left(N_{3}\right)$ : points of all groups. This relation is determined by $\sum_{i=1}^{4} g_{A}\left(N_{i}\right)=0$.

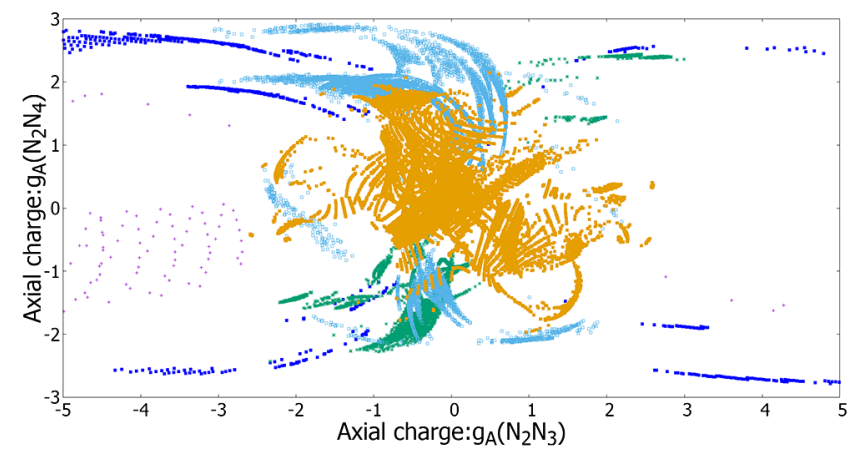

FIG. 4. Predicted values of $g_{A}\left(N_{2} N_{3}\right)$ and $g_{A}\left(N_{2} N_{4}\right)$. 


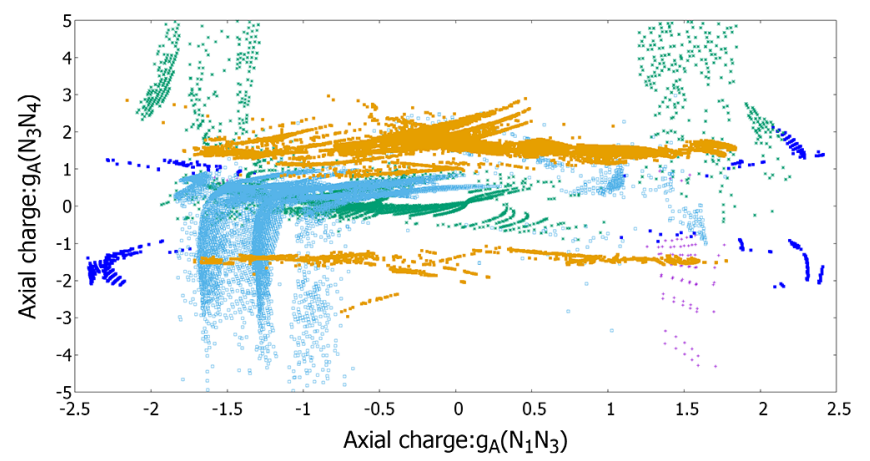

FIG. 5. Predicted values of $g_{A}\left(N_{3} N_{4}\right)$ and $g_{A}\left(N_{1} N_{3}\right)$.

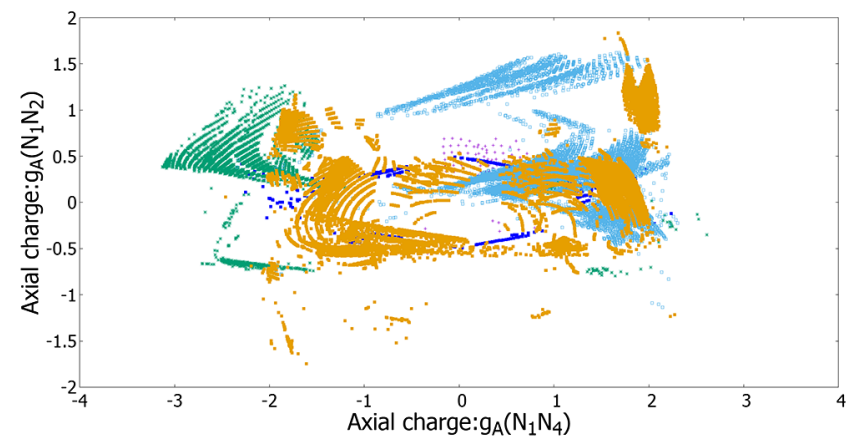

FIG. 6. Predicted values of $g_{A}\left(N_{1} N_{2}\right)$ and $g_{A}\left(N_{1} N_{4}\right)$ : There are no points of group 3 in the vicinity of $\left|g_{A}\left(N_{1} N_{4}\right)\right| \sim 0$. Values of group 1, 2, 4 and 5 are mixed there.

the lattice analysis, the axial charge of $N(1440)$ is negative. We compare these values with other models. In Ref. [15] the sum of diagonal axial charges is about 3.2 and that of $N(1440)$ is positive $\left[g_{A}(N(1440)) \sim 1.2\right]$. On the other hand, the axial charge of $N(1440)$ is negative $\left[-1 \leq g_{A}(N(1440)) \leq-0.5\right]$ in Ref. [14]. In Fig. 4 we plot predicted values of $g_{A}\left(N_{2} N_{3}\right)$ and $g_{A}\left(N_{2} N_{4}\right)$. This shows that $\left|g_{A}\left(N_{2} N_{3}\right)\right|$ of group 1 and group 2 is always larger than 2 and 0.5 , respectively. On the other hand, $\left|g_{A}\left(N_{2} N_{4}\right)\right|$ of group 1 is smaller than 2 and that of group 2 is above 1 . We see that group 1 is able to be distinguished from other groups. Predicted values of $g_{A}\left(N_{3} N_{4}\right)$ and $g_{A}\left(N_{1} N_{3}\right)$ are plotted in Fig. 5. We note that $\left|g_{A}\left(N_{1} N_{3}\right)\right|$ belonging to group 1 and group 2 are larger than 1, and that $\left|g_{A}\left(N_{3} N_{4}\right)\right|$ of group 2 and group 5 lies between 1 and 3 and that of group 1 is above 1 . In particular, $\left|g_{A}\left(N_{3} N_{4}\right)\right| \sim 2$ in group 5. We plot the values of $g_{A}\left(N_{1} N_{2}\right)$ and $g_{A}\left(N_{1} N_{4}\right)$ in Fig. 6. From Fig. 6, we can find that $\left|g_{A}\left(N_{1} N_{2}\right)\right|$ belonging to group 1 (purple + symbols) and group 2 (blue - symbols) are no larger than 1, while that belonging to group 3 (light green $\times$ symbols) and group 4 light blue $*$ symbols are below 1.5. $\left|g_{A}\left(N_{1} N_{4}\right)\right|$ belonging to group 3 (light green $\times$ symbols) are larger than 0.5 .

We summarize typical predicted values of transition axial charges and the range of transition axial charges in Table IV.

\section{B. Change of nucleon masses}

We plot the dependences of nucleon masses on the value of the VEV for groups 1-4 in Fig. 7 and those for group 5 in Fig. 8 for some choices of the chiral invariant masses, $m_{0}^{(1)}$ and $m_{0}^{(2)}$. Figure 7 shows that nucleons masses are

TABLE IV. Typical predicted values of axial charges. Rows indicated by $: j:(j=1, \ldots, 5)$ show that the absolute value of axial charge lies in the range shown in the rows.

\begin{tabular}{lccccccc}
\hline \hline Group & $\left(m_{0}^{(1)}, m_{0}^{(2)}\right)$ & $g_{A}\left(N_{1} N_{2}\right)$ & $g_{A}\left(N_{1} N_{3}\right)$ & $g_{A}\left(N_{1} N_{4}\right)$ & $g_{A}\left(N_{2} N_{3}\right)$ & $g_{A}\left(N_{2} N_{4}\right)$ & $g_{A}\left(N_{3} N_{4}\right)$ \\
\hline 1 & $(5,100)$ & 0.689 & 1.707 & -0.159 & -4.512 & -0.197 & -4.303 \\
1 & $(40,95)$ & -0.505 & -1.353 & 0.417 & -2.859 & 1.310 & 0.804 \\
$: 1:$ & $\cdots$ & $\left|g_{A}\right|<1$ & $1<\left|g_{A}\right|<2$ & $\left|g_{A}\right|<1$ & $\left|g_{A}\right|>2.5$ & $\left|g_{A}\right|<2$ & $\left|g_{A}\right|>0.5$ \\
2 & $(510,540)$ & 0.449 & -2.224 & 0.247 & -4.644 & 2.614 & -1.979 \\
2 & $(645,715)$ & -0.434 & 1.868 & -1.132 & -1.039 & 2.204 & -0.921 \\
2 & $(790,855)$ & 0.252 & -1.912 & -1.306 & -1.294 & -1.614 & 1.238 \\
$: 2:$ & $\cdots$ & $\left|g_{A}\right|<1$ & $\left|g_{A}\right|>1$ & $\left|g_{A}\right|<2.5$ & $\left|g_{A}\right|>0.5$ & $\left|g_{A}\right|>1$ & $\left|g_{A}\right|>0.5$ \\
3 & $(400,175)$ & 0.906 & -1.197 & -1.697 & 0.935 & -1.394 & 1.561 \\
3 & $(695,330)$ & 0.206 & 1.549 & 0.888 & 2.825 & 2.394 & 4.95 \\
3 & $(940,620)$ & 0.203 & 1.963 & 1.597 & 1.197 & 1.324 & -0.242 \\
$: 3:$ & $\cdots$ & $\left|g_{A}\right|<1.5$ & $\left|g_{A}\right|<2.5$ & $\left|g_{A}\right|<4$ & $\left|g_{A}\right|<3$ & $\left|g_{A}\right|<2.5$ & $g_{A}>-1$ \\
4 & $(365,685)$ & 1.328 & -1.694 & 0.175 & -2.219 & 2.028 & -1.419 \\
4 & $(650,945)$ & -0.099 & -0.149 & 1.959 & -0.534 & -1.386 & 0.795 \\
4 & $(1070,1150)$ & -0.755 & -0.303 & 2.136 & -0.293 & -0.159 & 1.714 \\
$: 4:$ & $\cdots$ & $\left|g_{A}\right|<1.5$ & $\left|g_{A}\right|<2$ & $g_{A}<2.5$ & $\left|g_{A}\right|<3.5$ & $\left|g_{A}\right|<3$ & $g_{A}<2$ \\
5 & $(1070,50)$ & -0.514 & -1.350 & -1.454 & 0.075 & -0.548 & -1.415 \\
5 & $(1085,500)$ & 0.713 & 1.210 & -1.599 & -0.058 & 0.107 & 1.492 \\
5 & $(1210,1050)$ & -0.295 & 0.175 & 1.788 & -0.245 & 0.116 & 1.867 \\
$: 5:$ & $\cdots$ & $\left|g_{A}\right|<2$ & $\left|g_{A}\right|<2.5$ & $\left|g_{A}\right|<2.5$ & $\left|g_{A}\right|<3$ & $\left|g_{A}\right|<2$ & $1<\left|g_{A}\right|<3$ \\
\hline \hline
\end{tabular}




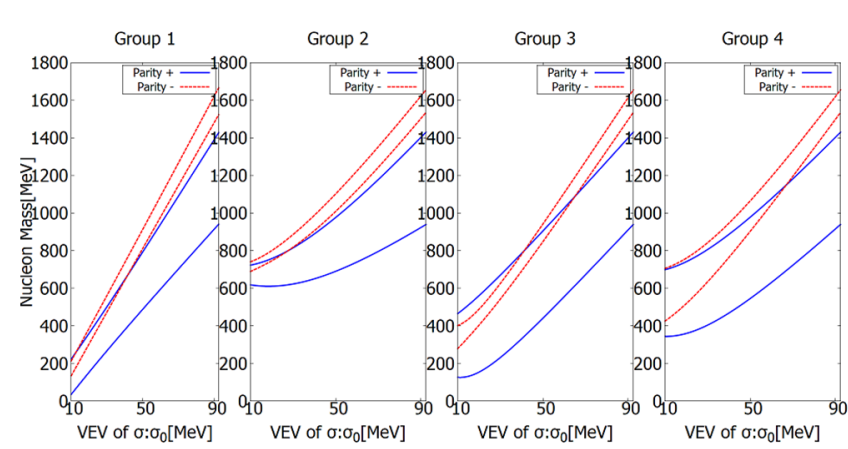

FIG. 7. Dependences of nucleon masses on the VEV of $\sigma$ for groups $1-4$.Values of chiral invariant masses used are $\left(m_{0}^{(1)}\right.$, $\left.m_{0}^{(2)}\right)=(40 \mathrm{MeV}, 95 \mathrm{MeV}) \quad($ group 1$),\left(m_{0}^{(1)}, m_{0}^{(2)}\right)=(645 \mathrm{MeV}$, $715 \mathrm{MeV}) \quad($ group 2$), \quad\left(m_{0}^{(1)}, m_{0}^{(2)}\right)=(400 \mathrm{MeV}, 175 \mathrm{MeV})$ (group 3) and $\left(m_{0}^{(1)}, m_{0}^{(2)}\right)=(365 \mathrm{MeV}, 685 \mathrm{MeV})$ (group 4).

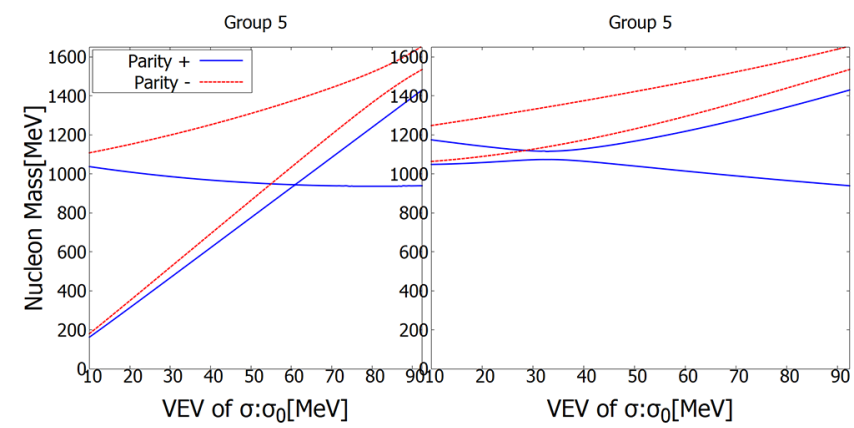

FIG. 8. Dependences of nucleon masses on the VEV of $\sigma$ in group 5 for $\left(m_{0}^{(1)}, m_{0}^{(2)}\right)=(1070 \mathrm{MeV}, 50 \mathrm{MeV})$ (left figure) and (1210 MeV, $1050 \mathrm{MeV}$ ) (right figure).

decreased as $\sigma_{0}$ is decreased in groups 1,2, 3 and 4, if chiral invariant masses are smaller than the mass of $N(939)$. We note that, for some parameter choices in group 4 , the mass of the ground state nucleon is increased as $\sigma_{0}$ is decreased.

In the case of group 5 shown in Fig. 8, the value of $m_{0}^{(1)}$ is about $1000 \mathrm{MeV}$, while $m_{0}^{(2)}$ takes values in the wide range. The left panel of Fig. $8\left[\left(m_{0}^{(1)}, m_{0}^{(2)}\right)=(1070 \mathrm{MeV}\right.$, $50 \mathrm{MeV})$ ] shows that the mass of the ground state is stable for $\sigma_{0}>60 \mathrm{MeV}$ and it decreased towards $m_{0}^{(2)}$ as $\sigma_{0}$ is decreased from $60 \mathrm{MeV}$. On the other hand, the right panel $[(1210 \mathrm{MeV}, 1050 \mathrm{MeV})]$ shows that all the masses are stable against the change or $\sigma_{0}$.

Since $\sigma_{0}$ is an order parameter of chiral symmetry, Figs. 7 and 8 show that nucleon masses are degenerated to chiral invariant masses when the chiral symmetry is restored in e.g., high temperature and/or density.

In Ref. [3], only one pair of $[(\mathbf{1}, \mathbf{2}) \oplus(\mathbf{2}, \mathbf{1})]$ representation is included and the chiral invariant mass is determined as $270 \mathrm{MeV}$. The dependence of the masses of $N(939)$ and $N(1535)$ on the VEV is consistent with the ones for group 3 and group 4 in Fig. 7. The dependences of four masses for minimum 1 and minimum 2 in Ref. [15] are similar to the one for group 3, while that for minimum 3 is to group 4 .

\section{SUMMARY AND DISCUSSIONS}

We introduced two types of nucleons belonging to $[(\mathbf{2}, \mathbf{1}) \oplus(\mathbf{1}, \mathbf{2})]$ and $[(\mathbf{2}, \mathbf{3}) \oplus(\mathbf{3}, \mathbf{2})]$ representations of the chiral $\mathrm{SU}(2)_{L} \otimes \mathrm{SU}(2)_{R}$ group together with their parity partners. We constructed an effective chiral Lagrangian based on the parity doublet structure. We fitted model parameters to the masses, decay widths and axial charges of $N(939), N(1440), N(1535)$ and $N(1650)$. Our results show that there are five groups of solutions which are separated by chiral invariant masses and mixing structure of nucleons. In group 1, both the chiral invariant masses, $m_{0}^{(1)}$ for $[(\mathbf{2}, \mathbf{1}) \oplus(\mathbf{1}, \mathbf{2})]$ and $m_{0}^{(2)}$ for $[(\mathbf{2}, \mathbf{3}) \oplus(\mathbf{3}, \mathbf{2})]$, are small as seen from Fig. 1. In this group, the ground state $N(939)$ is dominated by the $[(\mathbf{2}, \mathbf{3}) \oplus(\mathbf{3}, \mathbf{2})]$ component and its chiral partner is $N(1440)$. In group 5, on the other hand, the dominant component of $N(939)$ is $[(\mathbf{2}, \mathbf{1}) \oplus(\mathbf{1}, \mathbf{2})]$, whose chiral invariant mass is about $1000 \mathrm{MeV}$, and the chiral partner of $N(939)$ is a mixture of negative parity nucleons.

In the present analysis, the sum of four axial charges satisfies the sum rule in Eq. (47). When the axial charges of $N(939)$ and $N(1650)$ are fixed as we have done in this paper, the charges of $N(1440)$ and $N(1535)$ follow the relation as shown in Fig. 3.

We also gave predictions on transition axial charges. We expect that some groups can be excluded when some of them are determined by e.g., lattice analysis in the future. We note here that the $N(939)$ is not always dominated by $\eta_{1}$ which carries the axial charge $5 / 3$. The derivative interaction terms provide important contributions to axial charges in the present model, so that $N(939)$ is not dominated by $\eta_{1}$ in groups 3-5.

We also study the change of nucleon masses when the $\mathrm{VEV}$ of $\sigma, \sigma_{0}$, is changed. In groups $1-4$, all nucleon masses are decreased with decreasing $\sigma_{0}$ if two chiral invariant masses are smaller than the mass of $N(939)$ as shown in Fig. 7. In group 5, on the other hand, the behavior depends on the value of $m_{0}^{(2)}$ : For small $m_{0}^{(2)}$ the mass of $N(939)$ is stable for $\sigma_{0}>60 \mathrm{MeV}$ and it decreases toward $m_{0}^{(2)}$, while for large $m_{0}^{(2)}$ it is stable for all $\sigma_{0}$. This seems consistent with the lattice analysis in Refs. [17,18], which shows that, with increasing temperature, the mass of the positive parity nucleon mass is stable, while that of the negative parity nucleon mass decreased. From this, one might be able to exclude group 1 in which both the chiral invariant masses are small.

It would be useful to construct interpolating operators for e.g., the microscopic currents of four baryonic fields in terms of quarks for making a comparison with lattice results. We leave this for future work. 
The chiral representation of $[(\mathbf{2}, \mathbf{3}) \oplus(\mathbf{3}, \mathbf{2})]$ includes $\Delta$ baryon in addition to nucleons. For studying $\Delta$, we need to include $[(\mathbf{4}, \mathbf{1}) \oplus(\mathbf{1}, \mathbf{4})]$ representations which do not include nucleons. It is interesting to study $\Delta$ baryons by constructing a model including $[(\mathbf{4}, \mathbf{1}) \oplus(\mathbf{1}, \mathbf{4})]$ representations. [Study of the $\Delta$ baryon based on the parity doublet structure is done in e.g., Refs. [3,21-24].]

We can extend the model to three flavor case based on the $\mathrm{SU}(3)_{\mathrm{L}} \otimes \mathrm{SU}(3)_{\mathrm{R}}$ symmetry to study hyperons as done in Refs. $[6,7,10-16]$. The parity doublet structure can also be extended to the baryons including heavy quarks as done in Refs. [25-28].

It is interesting to construct nuclear matter in the present model and the density dependences of effective masses of nucleons as done in Refs. [23,29-42]. We leave this for future work.

\section{ACKNOWLEDGMENTS}

This work was supported partly by JSPS KAKENHI Grant No. JP16K05345.
[1] C. E. Detar and T. Kunihiro, Linear $\sigma$ model with parity doubling, Phys. Rev. D 39, 2805 (1989).

[2] D. Jido, Y. Nemoto, M. Oka, and A. Hosaka, Chiral symmetry for positive and negative parity nucleons, Nucl. Phys. A671, 471 (2000).

[3] D. Jido, M. Oka, and A. Hosaka, Chiral symmetry of baryons, Prog. Theor. Phys. 106, 873 (2001).

[4] S. Gallas, F. Giacosa, and D. H. Rischke, Vacuum phenomenology of the chiral partner of the nucleon in a linear sigma model with vector mesons, Phys. Rev. D 82, 014004 (2010).

[5] S. Gallas and F. Giacosa, Mirror versus naive assignment in chiral models for the nucleon, Int. J. Mod. Phys. A 29, 1450098 (2014).

[6] Y. Nemoto, D. Jido, M. Oka, and A. Hosaka, Decays of 1/2baryons in chiral effective theory, Phys. Rev. D 57, 4124 (1998).

[7] H. X. Chen, V. Dmitrasinovic, A. Hosaka, K. Nagata, and S. L. Zhu, Chiral properties of baryon fields with flavor SU(3) symmetry, Phys. Rev. D 78, 054021 (2008).

[8] V. Dmitrasinovic, A. Hosaka, and K. Nagata, Nucleon axial couplings and $[(1 / 2,0)+(0,1 / 2)]-[(1,1 / 2)+(1 / 2,1)]$ chiral multiplet mixing, Mod. Phys. Lett. A 25, 233 (2010).

[9] V. Dmitrasinovic, A. Hosaka, and K. Nagata, A Lagrangian for the chiral $(1 / 2,0) \oplus(0,1 / 2)$ quartet nucleon resonances, Int. J. Mod. Phys. E 19, 91 (2010).

[10] H. X. Chen, V. Dmitrasinovic, and A. Hosaka, Baryon fields with $U_{L}(3) \times U_{R}(3)$ chiral symmetry II: Axial currents of nucleons and hyperons, Phys. Rev. D 81, 054002 (2010).

[11] H. X. Chen, V. Dmitrasinovic, and A. Hosaka, Baryon fields with $U_{L}(3) \times U_{R}(3)$ chiral symmetry III: Interactions with chiral $(3, \overline{3}) \oplus(\overline{3}, 3)$ spinless mesons, Phys. Rev. D 83, 014015 (2011).

[12] H. X. Chen, V. Dmitrasinovic, and A. Hosaka, Baryons with $\mathrm{U}_{L}(3) \times \mathrm{U}_{R}(3)$ chiral symmetry IV: Interactions with chiral $(8,1)+(1,8)$ vector and axial-vector mesons and anomalous magnetic moments, Phys. Rev. C 85, 055205 (2012).

[13] V. Dmitrasinovic, H. X. Chen, and A. Hosaka, Baryon fields with $\mathrm{U}_{L}(3) \times \mathrm{U}_{R}(3)$ chiral symmetry. V. Pion-nucleon and kaon-nucleon $\Sigma$ terms, Phys. Rev. C 93, 065208 (2016).
[14] H. Nishihara and M. Harada, Extended Goldberger-Treiman relation in a three-flavor parity doublet model, Phys. Rev. D 92, 054022 (2015).

[15] L. Olbrich, M. Zètènyi, F. Giacosa, and D. H. Rischke, Three-flavor chiral effective model with four baryonic multiplets within the mirror assignment, Phys. Rev. D 93, 034021 (2016).

[16] L. Olbrich, M. Zètènyi, F. Giacosa, and D. H. Rischke, Influence of the axial anomaly on the decay $N(1535) \rightarrow N \eta$, Phys. Rev. D 97, 014007 (2018).

[17] G. Aarts, C. Allton, S. Hands, B. Jäger, C. Praki, and J. I. Skullerud, Nucleons and parity doubling across the deconfinement transition, Phys. Rev. D 92, 014503 (2015).

[18] G. Aarts, C. Allton, D. De Boni, S. Hands, B. Jäger, C. Praki, and J. I. Skullerud, Light baryons below and above the deconfinement transition: Medium effects and parity doubling, J. High Energy Phys. 06 (2017) 034.

[19] M. Tanabashi et al. (Particle Data Group), Review of particle physics, Phys. Rev. D 98, 030001 (2018).

[20] T. T. Takahashi and T. Kunihiro, Axial charges of $N(1535)$ and $N(1650)$ in lattice QCD with two flavors of dynamical quarks, Phys. Rev. D 78, 011503 (2008).

[21] D. Jido, T. Hatsuda, and T. Kunihiro, Chiral Symmetry Realization for Even Parity and Odd Parity Baryon Resonances, Phys. Rev. Lett. 84, 3252 (2000).

[22] K. Nagata, A. Hosaka, and V. Dmitrasinovic, $\pi N$ and $\pi \pi N$ Couplings of the $\Delta(1232)$ and its Chiral Partners, Phys. Rev. Lett. 101, 092001 (2008).

[23] Y. Takeda, Y. Kim, and M. Harada, Catalysis of partial chiral symmetry restoration by $\Delta$ matter, Phys. Rev. C 97, 065202 (2018).

[24] P. Bicudo, M. Cardoso, F. J. Llanes-Estrada, and T. Van Cauteren, Mapping chiral symmetry breaking in the excited baryon spectrum, Phys. Rev. D 94, 054006 (2016).

[25] Y. L. Ma and M. Harada, Doubly heavy baryons with chiral partner structure, Phys. Lett. B 748, 463 (2015).

[26] Y. L. Ma and M. Harada, Degeneracy of doubly heavy baryons from heavy quark symmetry, Phys. Lett. B 754, 125 (2016). 
[27] Y. L. Ma and M. Harada, Chiral partner structure of doubly heavy baryons with heavy quark spin-flavor symmetry, J. Phys. G 45, 075006 (2018).

[28] Y. Kawakami and M. Harada, Analysis of $\Lambda_{c}(2595)$, $\Lambda_{c}(2625), \Lambda_{b}(5912), \Lambda_{b}(5920)$ based on a chiral partner structure, Phys. Rev. D 97, 114024 (2018).

[29] T. Hatsuda and M. Prakash, Parity doubling of the nucleon and first order chiral transition in dense matter, Phys. Lett. B 224, 11 (1989).

[30] D. Zschiesche, L. Tolos, J. Schaffner-Bielich, and R. D. Pisarski, Cold, dense nuclear matter in a $\mathrm{SU}(2)$ parity doublet model, Phys. Rev. C 75, 055202 (2007).

[31] V. Dexheimer, S. Schramm, and D. Zschiesche, Nuclear matter and neutron stars in a parity doublet model, Phys. Rev. C 77, 025803 (2008).

[32] V. Dexheimer, G. Pagliara, L. Tolos, J. Schaffner-Bielich, and S. Schramm, Neutron stars within the SU(2) parity doublet model, Eur. Phys. J. A 38, 105 (2008).

[33] C. Sasaki and I. Mishustin, Thermodynamics of dense hadronic matter in a parity doublet model, Phys. Rev. C 82, 035204 (2010).

[34] S. Gallas, F. Giacosa, and G. Pagliara, Nuclear matter within a dilatation-invariant parity doublet model: The role of the tetraquark at nonzero density, Nucl. Phys. A872, 13 (2011).
[35] J. Steinheimer, S. Schramm, and H. Stocker, Hadronic $\mathrm{SU}(3)$ parity doublet model for dense matter, its extension to quarks and the strange equation of state, Phys. Rev. C 84, 045208 (2011).

[36] V. Dexheimer, J. Steinheimer, R. Negreiros, and S. Schramm, Hybrid stars in an SU(3) parity doublet model, Phys. Rev. C 87, 015804 (2013).

[37] S. Benic, I. Mishustin, and C. Sasaki, Effective model for the QCD phase transitions at finite baryon density, Phys. Rev. D 91, 125034 (2015).

[38] Y. Motohiro, Y. Kim, and M. Harada, Asymmetric nuclear matter in a parity doublet model with hidden local symmetry, Phys. Rev. C 92, 025201 (2015); Erratum, Phys. Rev. C 95, 059903(E) (2017).

[39] D. Suenaga, Examination of $N^{*}(1535)$ as a probe to observe the partial restoration of chiral symmetry in nuclear matter, Phys. Rev. C 97, 045203 (2018).

[40] Y. Takeda, H. Abuki, and M. Harada, Novel dual chiral density wave in nuclear matter based on a parity doublet structure, Phys. Rev. D 97, 094032 (2018).

[41] D. Suenaga, Spectral function for $\bar{D}_{0}^{*}\left(0^{+}\right)$meson in isospin asymmetric nuclear matter with chiral partner structure, arXiv:1805.01709.

[42] I. J. Shin, W. G. Paeng, M. Harada, and Y. Kim, Nuclear structure in parity doublet model, arXiv:1805.03402. 